\title{
Are we ready to include invasive cribriform and intraductal carcinoma into the prostate cancer grade grouping system?
}

\author{
Guang-Qian Xiao, Andy E. Sherrod \\ Department of Pathology, Keck School of Medicine, University of Southern California, Los Angeles, CA 90033, USA \\ Correspondence to: Guang-Qian Xiao, M.D. PhD. Department of Pathology, Keck School of Medicine, University of Southern California, Los \\ Angeles, CA 90033, USA. Email: guang-qian.xiao@med.usc.edu. \\ Comment on: van Leenders GJLH, Kweldam CF, Hollemans E, et al. Improved Prostate Cancer Biopsy Grading by Incorporation of Invasive \\ Cribriform and Intraductal Carcinoma in the 2014 Grade Groups. Eur Urol 2020;77:191-8.
}

Submitted Apr 02, 2020. Accepted for publication Apr 26, 2020.

doi: $10.21037 /$ tau-20-845

View this article at: http://dx.doi.org/10.21037/tau-20-845

Prostatic adenocarcinoma $(\mathrm{PCa})$ is one of the most heterogeneous tumors with great morphologic and biological diversity as well as variable clinical courses. It has been more than half a century since Dr. Gleason proposed the first PCa grading system. Over the years, even with emerging prognostic molecular biomarkers, Gleason grading based on the histomorphology is still the most powerful and widely used parameter in predicting the behavior of $\mathrm{PCa}$ as well as in determining the treatment modalities for PCa patients. Despite a huge success, its predictability for the clinical behavior of PCa is still limited, particularly for intermediate grade (grade groups 2 and 3) PCa. With growing knowledge and better understanding of PCa biology and its pathological features, the Gleason grading of PCa has been continuously evolving. To simplify and improve the group stratification of the PCa grading, a 5 Grade Group system has been developed based on modified original Gleason grading and is now incorporated into the 2016 WHO prostate cancer grading system (1). In the past decade, invasive cribriform PCa and intraductal carcinoma (IDC) have been increasingly recognized as independent adverse histomorphologic components in predicting the poor outcome of PCa (2-8), and the International Society of Urological Pathology has recently recommended to include the cribriform PCa and IDC in the routine pathology report.

In the recent article by van Leenders et al. published in European Urology, the authors incorporated the invasive cribriform PCa and IDC into the current grading group (GG) and analyzed this modified grade grouping (cGG) method in the prediction of the outcome of PCa patients (9). Their results have shown that the modified cGG system has better discriminative values for disease-specific survival as well as metastasis-free survival than the contemporary $\mathrm{PCa}$ grading group; especially, cGG significantly increases the eligibility for active surveillance. Overall it better predicts PCa outcome and might improve future treatment options as well.

The findings are appealing and promising. Although the underlying mechanisms for the aggressiveness of cribriform PCa are not fully understood, our recent study has shown overexpression of EGFR in the cribriform PCa in comparison with non-cribriform Gleason $4 \mathrm{PCa}$ (10). Inclusion of invasive cribriform morphology in the grade group may additionally benefit patients for potential future treatment with the available EGFR inhibitors (e.g., Cetuximab, Gefitinib and Erlotinib) as well. Nevertheless, besides the need of further validation by independent studies, there are a few issues we raise regarding this proposed modified grade grouping.

\section{The potential impact of Gleason 4 non-cribriform element might be overlooked}

The authors demonstrated that downgrading of Gleason 3 + non-cribriform Gleason 4 (GG2) to cGG1 increased the eligibility for active surveillance. The clinical consequence is still uncertain at this moment. The non-cribriform Gleason $4 \mathrm{PCa}$ is also a group of patterns with morphology varying from ill-formed glands to variably sized fused 
glands. Some of the fused glands, to some extent, may be morphologically close to cribriform PCa. At present, the biologic difference between fused glands and cribriform is not yet known and it is unclear whether or not these noncribriform Gleason 4 PCa variants behave differently. It has been known that the percentage of Gleason pattern 4 is also of prognostic value for PCa outcome (11), especially when the percentage/absolute volume of the non-cribriform Gleason 4 is at the high end of the $50 \%$ cutoff in GG2 disease. Although the authors did not find additive predictive value of the percentage of the Gleason pattern 4 , as the authors mentioned it could have been caused by the way it was analyzed. Loosening of the stringency and over downgrading of the GG2 to cGG1 for surveillance eligibility might risk a subset of the patients with $\mathrm{PCa}$ of aggressive potential for disease progression if active surveillance is selected for them. One of the cautious options to avoid this potential adverse effect is to subgroup the cGG1 into "cGG1a" for Gleason 3+3 (GG1) and "cGG1b" for Gleason 3+noncribriform Gleason 4 (noncribriform GG2). Nevertheless, the benefit for the shift of morphologic surveillance criteria by the proposed cGG requires further assessment.

\section{The potential impact of the morphologic variants of invasive cribriform PCa might need to be considered}

Morphologically, the cribriform pattern of invasive PCa can be further classified into glomeruloid (intraglandular cribriform) and conventional cribriform. It has been shown that the prognosis of invasive $\mathrm{PCa}$ with conventional cribriform pattern is worse than that of $\mathrm{PCa}$ with glomeruloid cribriform pattern $(12,13)$. Conventional nonglomeruloid cribriform PCa can also be divided into PCa of small cribriform, defined by the size equal or smaller than adjacent benign prostatic glands and without tangible expansion, and PCa of large expansible cribriform. We have recently demonstrated that lymphovascular invasion and lymph node metastasis are strongly associated with small cribriform PCa (14). On the other hand, large cribriform lesions, although often being called invasive $\mathrm{PCa}$, not infrequently contain variable basal cells in the periphery if basal cell immunohistochemistry is performed, and, are actually non-invasive. Although these large cribriform lesions with basal cells could represent intraductal carcinomas, at least some of them could be precursor lesions (such as high grade PINs or carcinomas in situ) (15). It is conceivable that invasive small cribriform $\mathrm{PCa}$ is more accessible to the blood or lymphatic stream than invasive large cribriform PCa. More recently, some researchers have found that formation of small cell clusters/ groups are essential for tumor cells to survive in blood or lymphatics as well as to initially establish tumor colonies at metastatic sites $(16,17)$. In addition, detection of groups/ clusters of tumor cells is found to signify a worse prognosis than dispersed tumor cells in the blood stream $(18,19)$. These findings also support the importance of the ability of tumor cells to retain cohesion and form a group in the success of migration and distant seeding. Given the different pathologic presentations for different cribriform morphologies, the variants of invasive cribriform PCa may need to be considered as well in the cGG grade grouping for improvement of its accuracy.

\section{Potential impact of the volume of cribriform PCa}

Reporting of the percentage of Gleason 4/5 pattern has recently been implemented. Percentage or absolute volume of invasive cribriform PCa might also be important. Although no specific study has been conducted to evaluate and correlate the impact of percentage/volume of invasive cribriform $\mathrm{PCa}$ on clinical outcome. Low percentage or only one or a few invasive foci of cribriform PCa should apparently have more favorable overall outcome than high percentage or abundant invasive cribriform PCa. Therefore, the percentage/volume of invasive cribriform $\mathrm{PCa}$ might also need to be considered.

\section{The intraductal carcinoma mystery}

IDC is thought to represent intraductal spread and cancerization of preexisting ducts and/or acini by invasive carcinoma and is typically associated with high-grade and high-stage PCa $(7,8)$. Definitive therapy or immediate rebiopsy is currently recommended if IDC is identified in a biopsy specimen. IDC is morphologically characterized by solid, dense cribriform, comedonecrosis, or a loose cribriform or micropapillary pattern with marked nuclear atypia $(8,20)$. Despite the delineated morphologic criteria, in practice, distinction between noninvasive atypical cribriform lesion or atypical intraductal proliferation and IDC might not be so straightforward, and a gray zone exists with high inter-observer variability. In addition, recently we have shown that IDCs do not necessarily display the aforementioned cytological or architectural morphology 
of classic IDCs (15). They can present with features of low-grade cytology as well as variable architectures (15). Furthermore, it is well known that no invasive carcinoma is found in at least $10 \%$ of the cases containing lesions with IDC morphology $(7,21)$. This fact indicates that, at least some, if not most, of the IDCs are actually precursor lesions or carcinomas in situ rather than retrograde spreads of surrounding invasive $\mathrm{PCa}$, which also seems to be endorsed by our study (15). So far, there are no definitive morphologic features, immunohistochemical or even molecular biomarkers that distinguish IDCs from the precursor lesions of PCa. Given the current relative uncertainty of the diagnosis of IDC, its weight in grade grouping may be variable and subject to inter-observer variation. Further studies and better definition of the criteria for this entity are warranted.

In summary, the authors presented a very interesting longitudinal study with a sound proposal for the improvement of PCa grade group. Future validation and comprehensive consideration is still needed. In the near future, we anticipate an improvement in the stratification of PCa patients by incorporating not only morphologic parameters but also potential molecular biomarkers, including genomic signatures.

\section{Acknowledgments}

Funding: None.

\section{Footnote}

Provenance and Peer review: This article was commissioned by the editorial office, Translational Andrology and Urology. The article did not undergo external peer review.

Conflicts of Interest: Both authors have completed the ICMJE uniform disclosure form (available at http://dx.doi. org/10.21037/tau-20-845). Both authors have no conflicts of interest to declare.

Ethical Statement: The authors are accountable for all aspects of the work in ensuring that questions related to the accuracy or integrity of any part of the work are appropriately investigated and resolved.

Open Access Statement: This is an Open Access article distributed in accordance with the Creative Commons Attribution-NonCommercial-NoDerivs 4.0 International
License (CC BY-NC-ND 4.0), which permits the noncommercial replication and distribution of the article with the strict proviso that no changes or edits are made and the original work is properly cited (including links to both the formal publication through the relevant DOI and the license). See: https://creativecommons.org/licenses/by-nc-nd/4.0/.

\section{References}

1. Epstein JI, Zelefsky MJ, Sjoberg DD, et al. A Contemporary Prostate Cancer Grading System: A Validated Alternative to the Gleason Score. Eur Urol 2016;69:428-35.

2. Truong M, Frye T, Messing E, et al. Historical and contemporary perspectives on cribriform morphology in prostate cancer. Nat Rev Urol 2018;15:475-82.

3. Sarbay BC, Kir G, Topal, CS, et al. Significance of the cribriform pattern in prostatic adenocarcinomas. Pathol Res Pract 2014;210:554-7.

4. Kir G, Sarbay, BC, Gumus E, et al. The association of the cribriform pattern with outcome for prostatic adenocarcinomas. Pathol Res Pract 2014;210:640-4.

5. McKenney JK, Wei W, Hawley S, et al. Histologic grading of prostatic adenocarcinoma can be further optimized: analysis of the relative prognostic strength of individual architectural patterns in 1275 patients from the Canary retrospective cohort. Am J Surg Pathol 2016;40:1439-56.

6. Siadat, F, Sykes J, Zlotta AR, et al. Not all Gleason pattern 4 prostate cancers are created equal: A study of latent prostatic carcinomas in a cystoprostatectomy and autopsy series. Prostate 2015;75:1277-84.

7. Robinson BD, Epstein JI. Intraductal carcinoma of the prostate without invasive carcinoma on needle biopsy: emphasis on radical prostatectomy findings. J Urol 2010;184:1328-33.

8. Humphrey PA, Moch H, Cubilla AL, et al. The 2016 WHO Classification of Tumours of the Urinary System and Male Genital Organs-Part B: Prostate and Bladder Tumours. Eur Urol 2016;70:106-19.

9. van Leenders GJLH, Kweldam CF, Hollemans E, et al. Improved Prostate Cancer Biopsy Grading by Incorporation of Invasive Cribriform and Intraductal Carcinoma in the 2014 Grade Groups. Eur Urol 2020;77:191-8.

10. Xiao GQ, Nguyen E, Unger PD, et al. Comparative expression of immunohistochemical biomarkers in cribriform and pattern 4 non-cribriform prostatic adenocarcinoma. Exp Mol Pathol 2020;114:104400. 
11. Choy B, Pearce SM, Anderson BB, et al. Prognostic Significance of Percentage and Architectural Types of Contemporary Gleason Pattern 4 Prostate Cancer in Radical Prostatectomy. Am J Surg Pathol 2016;40:1400-6.

12. Iczkowski KA, Paner GP, Van der Kwast T. The New Realization About Cribriform Prostate Cancer. Adv Anat Pathol 2018;25:31-7.

13. Hollemans E, Verhoef EI, Bangma CH, et al. Clinicopathological characteristics of glomeruloid architecture in prostate cancer. Mod Pathol 2020;33:1618-25.

14. Chen ZS, Suen C, Unger P, Xiao GQ. Lymphovascular Invasion and Lymph Node Metastasis is Highly Associated with Small Cribriform Pattern of Invasive Prostatic Carcinoma. USCAP 2020 Abstracts, Modern Pathology 2020;33:873-4.

15. Xiao GQ, Golestani R, Pham H, et al. Stratification of Atypical Intraepithelial Prostatic Lesions Based on Basal Cell and Architectural Patterns. Am J Clin Pathol 2020;153:407-16.

16. Keller L, Pantel K. Unravelling tumour heterogeneity by

Cite this article as: Xiao GQ, Sherrod AE. Are we ready to include invasive cribriform and intraductal carcinoma into the prostate cancer grade grouping system? Transl Androl Urol 2020;9(5):2292-2295. doi:10.21037/tau-20-845 single-cell profiling of circulating tumour cells. Nat Rev Cancer 2019;19:553-67.

17. Gkountela S, Castro-Giner F, Szczerba BM, et al. Circulating Tumor Cell Clustering Shapes DNA Methylation to Enable Metastasis Seeding. Cell 2019;176:98-112.

18. Wang C, Mu Z, Chervoneva I, et al. Longitudinally collected CTCs and CTC-clusters and clinical outcomes of metastatic breast cancer. Breast Cancer Res. Treat 2017;161:83-94.

19. Aceto N, Bardia A, Miyamoto DT, et al. Circulating tumor cell clusters are oligoclonal precursors of breast cancer metastasis. Cell 2014;158:1110-22.

20. Guo CC, Epstein JI. Intraductal carcinoma of the prostate on needle biopsy: histologic features and clinical significance. Mod Pathol 2006;19:1528-35.

21. Miyai K, Divatia MK, Shen SS, et al. Heterogeneous clinicopathological features of intraductal carcinoma of the prostate: a comparison between "precursorlike" and "regular type" lesions. Int J Clin Exp Pathol 2014;7:2518-26. 